\title{
Five-Loop Critical Temperature Shift in Weakly Interacting Homogeneous Bose-Einstein Condensate
}

\author{
H. Kleinert \\ Institut für Theoretische Physik, \\ Freie Universität Berlin, Arnimallee 14, 14195 Berlin, Germany
}

\begin{abstract}
Using variational perturbation theory, we calculate the shift in the critical temperature $T_{c}$ up to five loops to lowest order in the scattering length $a$ and find $\Delta T_{c} / T_{c}^{(0)} \approx(1.14 \pm 0.11) a n^{1 / 3}$, where $n$ is the particle density. Our result is lower than the latest Monte Carlo result (1.32 \pm 0.02$) a n^{1 / 3}$.
\end{abstract}

PACS numbers: 03.75.Fi, 05.30.Jp, 12.38.Cy, 11.10.Wx

The effect of a small repulsive interaction upon the critical temperature $T_{c}$ of a Bose-Einstein condensate (BEC) has been a matter of controversy for many years, and the various results are converging only very slowly towards a common answer. Here we want to contribute to the ongoing discussion with a further result obtained from a resummation of Feynman diagrams up to five loops.

The interacting Bose-Einstein condensate is described by the euclidean action

$$
\mathcal{A}_{E}=\int_{0}^{\beta} d \tau \int d^{3} x\left\{\psi^{*}(\mathbf{x}, \tau)\left(\partial_{\tau}-\frac{1}{2 M} \nabla^{2}\right) \psi(\mathbf{x}, \tau)-\mu \psi^{*}(\mathbf{x}, \tau) \psi(\mathbf{x}, \tau)+\frac{2 \pi a}{M}\left[\psi(\mathbf{x}, \tau) \psi^{*}(\mathbf{x}, \tau)\right]^{2}\right\},
$$

where $M$ is the mass of the bosons, $\beta$ the inverse temperature in natural units with $\hbar=k_{B}=1, a$ is the $s$-wave scattering length, and $\mu$ the chemical potential. The free system has a transition temperature

$$
T_{c}^{(0)}=\frac{2 \pi}{M}\left[\frac{n}{\zeta(3 / 2)}\right]^{\frac{2}{3}},
$$

where $n$ is the particle density. A small relative shift of $T_{c}$ with respect to $T_{c}^{(0)}$ can be calculated from the general formula

$$
\frac{\Delta T_{c}}{T_{c}^{(0)}}=-\frac{2}{3} \frac{\Delta n}{n^{(0)}}
$$

where $n^{(0)}$ is the particle density in the free condensate and $\Delta n$ its change at $T_{c}$ caused by the small interaction. For small $a$, this behaves like [1, 2]

$$
\frac{\Delta T_{c}}{T_{c}^{(0)}}=c_{1} a n^{1 / 3}+\left[c_{2}^{\prime} \ln \left(a n^{1 / 3}\right)+c_{2}\right] a^{2} n^{2 / 3}+\mathcal{O}\left(a^{3} n\right)
$$

where $c_{2}^{\prime}=-64 \pi \zeta(1 / 2) / 3 \zeta(3 / 2)^{5 / 3} \simeq 19.7518$ can be calculated perturbatively, whereas $c_{1}$ and $c_{2}$ require nonperturbative techniques since infrared divergences at $T_{c}$ make them basically strong-coupling results. The standard technique to reach this regime is based on a resummation of perturbation expansions using the renormalization group [3, 4], first applied in this context by Ref. 6]. Recently, however, it has been shown by calculating the best known critical exponent $\alpha$ of superfluid helium from Satellite experiments 7] that the accuracy of strong-coupling results can be surpassed by much simpler variational perturbation theory [4, 8, 9].

Up to now, $c_{2}$ has been inferred only from Monte Carlo data to be $c_{2} \approx 75.7 \pm 0.4$. In order to find the leading coefficient $c_{1}$, one may take advantage of an important simplification due to the fact that $\Delta n$ can be calculated from the classical limit of the field theory, which is governed by the three-dimensional action

$$
\mathcal{A}_{3 d}=\beta \int d^{3} x\left\{\psi_{0}^{*}(\mathbf{x})\left(-\frac{1}{2 M} \nabla^{2}-\mu\right) \psi_{0}(\mathbf{x})+\frac{2 \pi a}{M}\left[\psi_{0}^{*}(\mathbf{x}) \psi_{0}(\mathbf{x})\right]^{2}\right\} .
$$

This is a special case $N=2$ of the more general $\mathrm{O}(N)$-invariant $\phi^{4}$ field theory

$$
\mathcal{A}_{\phi}=\int d^{3} x\left[\frac{1}{2}|\nabla \phi|^{2}+\frac{1}{2} m^{2} \phi^{2}+\frac{u}{4 !}\left(\phi^{2}\right)^{2}\right],
$$


where the $N$-component field $\phi=\left(\phi_{1}, \phi_{2}, \ldots, \phi_{N}\right)$ is related to the original field $\psi$ for $N=2$ by $\psi(\mathbf{x})=$ $\sqrt{M T}\left[\phi_{1}(\mathbf{x})+i \phi_{2}(\mathbf{x})\right]$. The square mass is $m^{2}=-2 M \mu$, and the quartic coupling is $u=48 \pi a M T$. Using this relation, the shift of the critical temperature (3) can be found from the formula

$$
\frac{\Delta T_{c}}{T_{c}^{(0)}} \approx-\frac{2}{3} \frac{M T_{c}^{(0)}}{n}\left\langle\Delta \phi^{2}\right\rangle=-\frac{4 \pi}{3} \frac{\left(M T_{c}^{(0)}\right)^{2}}{n} 4 !\left\langle\frac{\Delta \phi^{2}}{u}\right\rangle a=-\frac{4 \pi}{3}(2 \pi)^{2} \frac{1}{[\zeta(3 / 2)]^{4 / 3}} 4 !\left\langle\frac{\Delta \phi^{2}}{u}\right\rangle a n^{1 / 3}
$$

corresponding in Eq. (41) to

$$
c_{1} \approx-1103.09\left\langle\frac{\Delta \phi^{2}}{u}\right\rangle
$$

The three-dimensional theory is superrenormalizable and requires only mass counterterms which shift the original bare mass $m$ to the renormalized mass $m_{r}$. A calculation of the Feynman diagrams in Fig. 11yields the following five-loop perturbation expansion for the expectation value $\left\langle\phi^{2} / u\right\rangle[10,11]$

$$
\begin{aligned}
\left\langle\frac{\phi^{2}}{u}\right\rangle= & F(u) \equiv-\frac{N}{4 \pi} \frac{m_{r}}{u}-a_{2} \frac{N(2+N)}{18(4 \pi)^{3}} \frac{u}{m_{r}}+a_{3} \frac{N\left(16+10 N+N^{2}\right)}{108(4 \pi)^{5}}\left(\frac{u}{m_{r}}\right)^{2} \\
& -\left[a_{41} \frac{N(2+N)^{2}}{324(4 \pi)^{7}}+a_{42} \frac{N\left(40+32 N+8 N^{2}+N^{3}\right)}{648(4 \pi)^{7}}+a_{43} \frac{N\left(44+32 N+5 N^{2}\right)}{324(4 \pi)^{7}}\right. \\
& \left.+a_{44} \frac{N(2+N)^{2}}{324(4 \pi)^{7}}+a_{45} \frac{N\left(44+32 N+5 N^{2}\right) u^{4}}{324 m_{r}^{3}(4 \pi)^{7}}\right]\left(\frac{u}{m_{r}}\right)^{3}+\ldots
\end{aligned}
$$

where $a_{2} \equiv \log (4 / 3) / 2 \approx 0.143841$ and the other constants are only known numerically [12]:

$$
a_{3}=0.642144, a_{41}=-0.115069, a_{42}=3.128107, a_{43}=1.63, a_{44}=-0.624638, a_{45}=2.39 \text {. }
$$

Writing the above expansion up to the $L$ th term as $F_{L}(u)=\Sigma_{l=-1}^{L} f_{l}\left(u / 4 \pi m_{r}\right)^{l}$, the expansion coefficients for the relevant number of components $N=2$ are [12]:

$$
f_{-1}=-126.651 \times 10^{-4}, \quad f_{0}=0, \quad f_{1}=-4.04837 \times 10^{-4}, \quad f_{2}=2.39701 \times 10^{-4}, \quad f_{3}=-1.80 \times 10^{-4} .
$$

We need the value of the series $F_{L}(u)$ in the critical limit $m_{r} \rightarrow 0$, which is obviously equivalent to the strong-coupling limit of $F_{L}(u)$. As mentioned above, this limit should be most accurately found with the help of variational perturbation theory [4, 8, 9].

If the series were of quantum mechanical origin, we could find this limit by applying the rules of naive variational perturbation theory [13]. We form the sequence of truncated expansions $F_{L}(u)$ for $1,2,3$ and replace each term

$$
\left(u / m_{r}\right)^{l} \rightarrow K^{l}[1-1]_{L-l}^{-l / 2}
$$

where the symbol $[1-1]_{k}^{r}$ is defined as the binomial expansion of $(1-1)^{r}$ truncated after the $k$ th term

$$
[1-1]_{k}^{r} \equiv \sum_{i=0}^{k}\left(\begin{array}{l}
r \\
i
\end{array}\right)(-1)^{i}=(-1)^{k}\left(\begin{array}{c}
r-1 \\
k
\end{array}\right)
$$

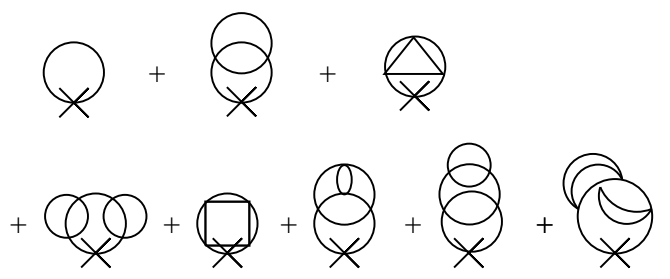

FIG. 1: Diagrams contributing to the expectation value $\left\langle\phi^{2}\right\rangle$. 
TABLE I: Trial functions for the naive quantum-mechanical variational perturbation expansion

$$
\begin{aligned}
& W_{1}^{\mathrm{QM}}=-0.0596831 K^{-1}-0.0000322159 K \\
& W_{2}^{\mathrm{QM}}=-0.0497359 K^{-1}-0.0000483239 K+1.5179210^{-6} K^{2}, \\
& W_{3}^{\mathrm{QM}}=-0.0435189 K^{-1}-0.0000604049 K+3.0358410^{-6} K^{2}-.90810^{-7} K^{3} .
\end{aligned}
$$

The resulting expressions must be optimized in the variational parameter $K$. They are listed in Table 【 The approximants $W_{1,2,3}^{\mathrm{QM}}$ have extrema $W_{1,2,3}^{\mathrm{QMext}} \approx-0.00277,+0.00405,-0.0029$, corresponding, via (8), to $c_{1} \approx 3.059,-4.46,3.01$. These values have previously been obtained in Ref. 10] in a much more complicated way via a so-called $\delta$-expansion. Note the negative sign of the second approximation arising from the fact that an extremum exists only at negative $K$. According to our rules of variational perturbation theory one should, in this case, use the saddle point at positive $K$ which would yield $W_{2}^{\mathrm{QM}}=-0.00153$ corresponding to $c_{1} \approx 1.69$ rather than -4.46 , leading to the more reasonable approximation sequence $c_{1} \approx 3.059,1.69,3.01$, which shows no sign of convergence. In $W_{3}^{\mathrm{QM}}$, there is also a pair of complex extrema from which the authors of Ref. [10] extract the real part $\operatorname{Re} \tilde{W}_{3 \text { complex }}^{\mathrm{QM}} \approx-0.00134$ corresponding to $c_{1} \approx 1.48$, which they state as their final result. There is, however, no acceptable theoretical justification for such a choice [14].

This lack of convergence is not astonishing since naive quantum mechanical variational perturbation theory is inapplicable to field theory, contrary to ubiquitous statements in the literature [15]. A simple but essential modification is necessary to allow for the well-known fact that there are anomalous dimensions in the critical regime of fluctuating fields. This modification was discovered in Ref. [8] and tested by the fact it reproduces in $D=4-\epsilon$ dimensions exactly the known $\epsilon$ expansions of renormalization group theory [9]. In $D=3$ dimensions, it leads to the most accurate critical exponents so far (see in particular Chapters 19 and 20 in the textbook [4]).

The correct procedure goes as follows: We form the logarithmic derivative of the expansion (9):

$$
\beta(u) \equiv \frac{\partial \log F(u)}{\partial \log u}=-1+2 \frac{f_{1}}{f_{-1}}\left(\frac{u}{m_{r}}\right)^{2}+3 \frac{f_{2}}{f_{-1}}\left(\frac{u}{m_{r}}\right)^{3}+\left(4 \frac{f_{3}}{f_{-1}}-2 \frac{f_{1}^{2}}{f_{-1}^{2}}\right)\left(\frac{u}{m_{r}}\right)^{4}+\ldots .
$$

In order for $F(u)$ to go to a constant in the critical limit $m_{r} \rightarrow 0$, this function must go to zero in the strong-coupling limit $u \rightarrow \infty$. Writing the expansion as $\beta_{L}(u)=-1+\Sigma_{l=2}^{L} b_{l}\left(u / 4 \pi m_{r}\right)^{l}$, the coefficients are

$$
b_{2}=0.0639293, \quad b_{3}=-0.056778, \quad b_{4}=0.0548799 .
$$

The sums $\beta_{L}(u)$ have to be evaluated for $u \rightarrow \infty$ allowing for the universal anomalous dimension $\omega$ by which the physical observables of $\phi^{4}$-theories approach the scaling limit [3, 4]. The approach to the critical point $A+B\left(m_{r} / u\right)^{\omega^{\prime}}$ where $\omega^{\prime}=\omega /(1-\eta / 2)[16$. The exponent $\eta$ is the small anomalous dimension of the field while $\omega$ is related to the famous Wegner exponent [5] of renormalization group theory $\Delta \equiv \omega \nu$. Here it appears in the variational expression for the strong-coupling limit which is found $[8,9]$ by replacing $\left(u / m_{r}\right)^{l}$ by $K^{l}[1-1]_{L-l}^{-q l / 2}$, where $q \equiv 2 / \omega^{\prime}$. Thus we obtain the variational expressions

$$
\begin{aligned}
& W_{3}^{\beta}=-1+\left(\frac{2 f_{1}}{f_{-1}}+\frac{2 f_{1} q}{f_{-1}}\right) K^{2}+\frac{3 f_{2}}{f_{-1}} K^{3} \\
& W_{4}^{\beta}=-1+\left(\frac{2 f_{1}}{f_{-1}}+\frac{3 f_{1} q}{f_{-1}}+\frac{f_{1} q^{2}}{f_{-1}}\right) K^{2}+\left(\frac{3 f_{2}}{f_{-1}}+\frac{9 f_{2} q}{2 f_{-1}}\right) K^{3}+\left(\frac{-2 f_{1}^{2}}{f_{-1}{ }^{2}}+\frac{4 f_{3}}{f_{-1}}\right) K^{4}
\end{aligned}
$$

The first has a vanishing extremum at $\omega_{3}^{\prime}=0.592$, the second has neither an extremum nor a saddle point. However, a complex pair of extrema lies reasonably close to the real axis at $\omega_{4}^{\prime}=0.635 \pm 0.116$, whose real part is not far from the true exponent of approach $\omega_{\infty}^{\prime} \approx 0.81$ [3, 4], to which $\omega_{L}^{\prime}$ will converge for order $L \rightarrow \infty$ [8]. Given these $\omega^{\prime}$-values, we now form the variational expressions $W_{L}$ from $F_{L}$ by the replacement $\left(u / m_{r}\right)^{l} \rightarrow K^{l}[1-1]_{L-l}^{-q l / 2}$, which are

$$
\begin{aligned}
& W_{2}=f_{-1}\left(1-\frac{3}{4} q+\frac{1}{8} q^{2}\right) K^{-1}+f_{1} K, \\
& W_{3}=f_{-1}\left(1-\frac{11}{13} q+\frac{1}{4} q^{2}-\frac{1}{48} q^{3}\right) K^{-1}+f_{1}\left(1+\frac{q}{2}\right) K+f_{2} K^{2}, \\
& W_{4}=f_{-1}\left(1-\frac{25}{24} q+\frac{35}{96} q^{2}-\frac{5}{96} q^{3}+\frac{1}{384} q^{4}\right) K^{-1}+f_{1}\left(1+\frac{3}{4} q+\frac{1}{8} q^{2}\right) K+f_{2}(1+q) K^{2}+f_{3} K^{3} .
\end{aligned}
$$


The lowest function $W_{2}$ is optimized with the naive growth parameter $q=1$ since to this order no anomalous value can be determined from the zero of the beta function (14). The optimal result is $W_{2}^{\text {opt }}=$ $-\sqrt{\log [4 / 3] / 6} / 8 \pi^{2} \approx-0.00277$ corresponding to $c_{1} \equiv 3.06$. The next function $W_{3}$ is optimized with the above determined $q_{3}=2 / \omega_{3}^{\prime}$ and yields $W_{3}^{\text {opt }} \approx-0.000976$ corresponding to $c_{1} \equiv 1.078$. Although $\omega_{4}^{\prime}$ is not real we shall insert its real part into $W_{4}$ and find $W_{4}^{\text {opt }} \equiv-0.000957$ corresponding to $c_{1} \equiv 1.057$. The three values of $c_{1}$ for $\bar{L} \equiv L-1=1,2,3$ can well be fitted by a function $c_{1} \approx 1.053+2 / \bar{L}^{6}$ (see Fig. 2 ). Such a fit is suggested by the general large- $L$ behavior $a+b e^{-c \bar{L}^{1-\omega^{\prime}}}$ which was derived in Refs. [13]. Due to the smallness of $1-\omega^{\prime} \approx 0.2$, this can be replaced by $\approx a^{\prime}+b^{\prime} / \bar{L}^{s}$.

Alternatively, we may optimize the functions $W_{1,2,3}$ using the known precise value of $q_{\infty}=2 / \omega_{\infty}^{\prime} \approx$ $2 / 0.81$. Then $W_{2}$ turns out to have no optimum, whereas the others yield $W_{3,4}^{\text {opt }} \approx-0.000554,-0.000735$, corresponding via Eq. (8) to $c_{1}=0.580,0.773$. If these two values are fitted by the same inverse power of $\bar{L}$, we find $c_{1} \approx 0.83-14 / \bar{L}^{6}$. From the extrapolations to infinite order we estimate $c_{1, \infty} \approx 0.92 \pm 0.13$.

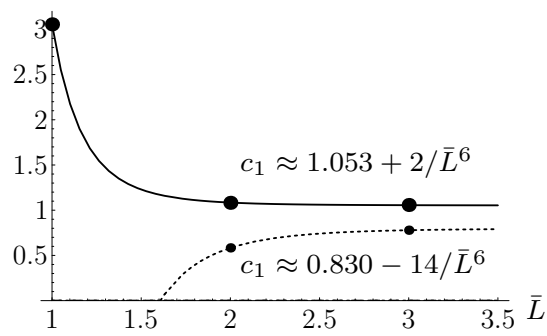

FIG. 2: The three approximants for $c_{1}$ plotted against the order of variational approximation $\bar{L} \equiv L-1=1,2,3$, and extrapolation to the infinite-order limit.

This result is to be compared with latest Monte Carlo data which estimate $c_{1} \approx 1.32 \pm 0.02[17$, 18]. Previous theoretical estimates are $c_{1} \approx 2.90[19], 2.33$ from a $1 / N$-expansion 20]), 1.71 from a next-toleading order in a $1 / N$-expansion [21], 3.059 from an inapplicable $\delta$-expansion [22] to three loops, and 1.48 from the same $\delta$-expansion to five loops, with a questionable evaluation at a complex extremum [10] and some wrong expansion coefficients (see 12]). Remarkably, our result lies close to the average between the latest and the first Monte Carlo result $c_{1} \approx 0.34 \pm 0.03$ in Ref. [23].

As a cross check of the reliability of our theory consider the result in the limit $N \rightarrow \infty$. Here we must drop the first term in the expansion (9) which vanishes at the critical point (but would diverge for $N \rightarrow \infty$ at finite $m_{r}$ ). The remaining expansion coefficients of $\left\langle\phi^{2} / u\right\rangle / N$ in powers of $N u / 4 \pi m_{r}$ are

$$
f_{1}=-6.3591710^{-4}, \quad f_{2}=4.731510^{-4}, \quad f_{3}=-3.8414610^{-4} \text {. }
$$

Using the $N \rightarrow \infty$ limit of $\omega^{\prime}$ which is equal to 1 implying $q=2$ in Eqs. (19) and (20), we obtain the two variational approximations

$$
W_{2}^{\infty}=-0.00127183 K+0.00047315 K^{2}, \quad W_{3}^{\infty}=-0.00190775 K+0.00141945 K^{2}-0.000384146 K^{3},
$$

whose optima yield the approximations $c_{1} \approx 1.886$ and 2.017 , converging rapidly towards the exact large- $N$ result 2.33 of Ref. [20], with a $10 \%$ error.

Numerically, the first two $1 / N$-corrections found from a fit to large- $N$ results obtained by using the known large- $N$ expression for $\omega^{\prime}=1-8\left(8 / 3 \pi^{2} N\right)+2\left(104 / 3-9 \pi^{2} / 2\right)\left(8 / 3 \pi^{2} N\right)^{2}$ [24] produce a finite- $N$ correction factor $\left(1-3.1 / N+30.3 / N^{2}+\ldots\right)$, to be compared with $(1-0.527 / N+\ldots)$ obtained in Ref. [21].

Since the large- $N$ results can only be obtained so well without the use of the first term we repeat the evaluations of the series at the physical value $N=2$ without the first term, where the variational expressions for $f$ are

$$
\begin{aligned}
& W_{2}=f_{1}\left(1+\frac{q}{2}\right) K+f_{2} K^{2} \\
& W_{3}=f_{1}\left(1+\frac{3}{4} q+\frac{1}{8} q^{2}\right) K+f_{2}(1+q) K^{2}+f_{3} K^{3} .
\end{aligned}
$$

The lowest order optimum lies now at $W_{2}^{\text {opt }}=-f_{1}^{2}(2+q)^{2} / 16 f_{2}^{2}$, yielding $c_{1} \equiv 0.942$ for the exact $q=2 / 0.81$. To next order, an optimal turning point of $W_{3}$ yields $c_{1} \approx 1.038$. 
At this order, we can derive a variational expression for the determination of $\omega^{\prime}$ using the analog of Eq. (14) which reads

$$
\beta(u) \equiv \frac{\partial \log F(u)}{\partial \log u}=1+\frac{f_{2}}{f_{1}} \frac{u}{m_{r}}+\left(2 \frac{f_{3}}{f_{1}}-\frac{f_{2}^{2}}{f_{1}^{2}}\right)\left(\frac{u}{m_{r}}\right)^{2}+\ldots .
$$

After the replacement (12) we find

$$
W_{3}^{\beta}=1+\frac{f_{2}(1+q / 2)}{f_{1}} K+\left(2 \frac{f_{3}}{f_{1}}-\frac{f_{2}^{2}}{f_{1}^{2}}\right) K^{2}+\ldots
$$

whose vanishing extremum determines $\omega^{\prime}=2 / q$ as being

$$
\omega_{3}^{\prime}=\left(2 \sqrt{2 f_{1} f_{3} / f_{2}^{2}-1}-1\right)^{-1} \approx 0.675
$$

leading to $c_{1} \approx 1.238$ from an optimal turning point of $W_{3}$. There are now too few points to perform an extrapolation to infinite order. From the average of the two highest-order results we obtain our final estimate: $c_{1} \approx 1.14 \pm 0.11$, such that the critical temperature shift is

$$
\frac{\Delta T_{c}}{T_{c}^{(0)}} \approx(1.14 \pm 0.11) a n^{1 / 3}
$$

This lies reasonably close to the Monte Carlo number $c_{1} \approx 1.32 \pm 0.02$.

Acknowledgment:

The author acknowledges useful communications with M. Pinto and R. Ramos on their work, as well as F. Nogueira and A. Pelster for many discussions. He also thanks B. Kastening for proofreading the paper, for confirming the approximate correctness of the Feynman integrals of Ref. 11] leading to the coefficients (10) rather than those of Ref. [10] (which are listed in [12] for comparison).

Note added in proof:

Since this paper appeared on the Los Alamos server last October, the Feynman diagrams where recalculated more accurately and extended to six loops in Ref. [25]. The more accurate $f_{3}$ has increased by about $6 \%$ to

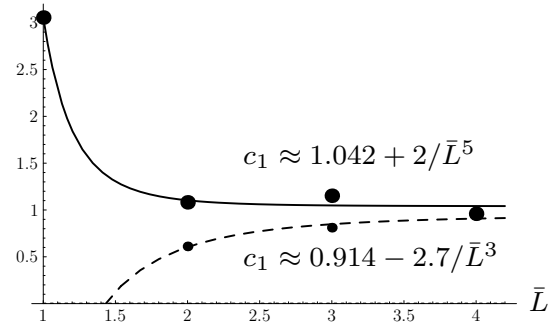

FIG. 3: The four approximants for $c_{1}$ plotted against the order of variational approximation $\bar{L} \equiv L-1=1,2,3,4$, and extrapolation to the infinite-order limit. The second-highest aproximations are $c_{1}=1.115$ and 0.797 for selfconsistent $\omega^{\prime}=0.745$ and exact $\omega^{\prime} \approx 0.81$, the highest aproximations are $c_{1}=0.959$ and 0.886 for self-consistent $\omega^{\prime}=0.745$ and exact $\omega^{\prime} \approx 0.81$, respectively. The latter dots cannot be distinguished.

$-1.92 \times 10^{-4}$, and the new expansion coefficient is $f_{4}=1.873 \times 10^{-4}$. Using the new numbers to perform a variational evaluation fits of the first type including the anomalous first term $f_{-1}$ we find the curves shown in Fig. [2] yielding $c_{1} \approx 0.98 \pm 0.06$, only slighly larger than than the previous five-loop number $0.92 \pm 0.13$. The upper curve is now associated with the self-consistent $\omega^{\prime}$-values $\omega_{3}^{\prime}=0.626$ and $\omega_{4}^{\prime}=0.745$, the last being almost equal to the exact value 0.81 .

The evaluation of the second type where the anomalous term is omitted yields now $c_{1}=0.942,1.056,1.120$, for $\omega^{\prime}=0.81$, and $c_{1}=1.409,1.384$ for the variationally determined $\omega_{3}^{\prime}=0.621$ and 0.638 , as shown in in Ref. 25]. Combining the last two results one obtains the larger value $c_{1} \equiv 1.23 \pm 0.12$, lying closer to the Monte Carlo number $c_{1} \approx 1.32 \pm 0.02$ than our five-loop result (27).

[1] M. Holzmann, G. Baym, J.-P. Blaizot and F. Laloë, Phys. Rev. Lett. 87, 120403 (2001). 
[2] P. Arnold, G. Moore and B. Tomásik, cond-mat/0107124

[3] J. Zinn-Justin, Quantum Field Theroy and Critical Phenomena (Oxford University Press, Oxford, England, 1996).

[4] H. Kleinert and V. Schulte-Frohlinde, Critical Properties of $\Phi^{4}$-Theories, World Scientific, Singapore 2001, pp. 1-487 (http://www.physik.fu-berlin.de/ kleinert/b8).

[5] F.J. Wegner, Phys. Rev. B 5, 4529 (1972); B 6, 1891 (1972).

6] M. Bijlsma and H. T. C. Stoof, Phys. Rev. A 54, 5085 (1996)

[7] J.A. Lipa, D.R. Swanson, J. Nissen, T.C.P. Chui, and U.E. Israelson, Phys. Rev. Lett. 76, 944 (1996). An improved best fit is given in Ref. [15] of J.A. Lipa, D.R. Swanson, J. Nissen, Z.K. Geng, P.R. Williamson, D.A. Stricker, T.C.P. Chui, U.E. Israelson, and M. Larson, Phys. Rev. Lett. 84, 4894 (2000). A recent reanalysis seems to confirm precisely the predicted value in the Abstract of the seven-loop paper in Ref. [8] (J.A. Lipa, private communication).

[8] H. Kleinert, Strong-Coupling Behavior of Phi ${ }^{4}$-Theories and Critical Exponents, Phys. Rev. D 57 , 2264 (1998); Addendum: Phys. Rev. D 58 , 107702 (1998) (cond-mat/9803268); Seven Loop Critical Exponents from StrongCoupling $\phi^{4}$-Theory in Three Dimensions, Phys. Rev. D 60 , 085001 (1999) (hep-th/9812197); Theory and Satellite Experiment on Critical Exponent alpha of Specific Heat in Superfluid Helium Phys. Lett. A 277, 205 (2000) (cond-mat/9906107).

[9] H. Kleinert, Strong-Coupling $\phi^{4}$-Theory in $4-\epsilon$ Dimensions, and Critical Exponent, Phys. Lett. B 434 , 74 (1998) (cond-mat/9801167); Critical Exponents without beta-Function, Phys. Lett. B 463, 69 (1999) (cond-mat/9906359).

[10] F. F. de Souza Cruz, M. B. Pinto and R. O. Ramos, Phys. Rev. A 65, 053613 (2002) (cond-mat/0112306).

[11] E. Braaten, and E. Radescu, (cond-mat/0206186).

[12] These are the results of [11]. They differ from those of Refs. 10] which are $a_{3}=0.644519, a_{41}=0.87339, a_{42}=$ $3.15905, a_{43}=1.70959, a_{44}=4.4411, a_{45}=2.37741$. The coefficients of the series (21) to be resummed differ mainly in the last term: $f_{-1}=-126.65110^{-4}, f_{0}=0, f_{1}=-4.0485710^{-4}, f_{2}=2.4058710^{-4}, f_{3}=-2.0684910^{-4}$.

[13] H. Kleinert, Path Integrals in Quantum Mechanics, Statistics and Polymer Physics, World Scientific, Singapore 1995, second extended edition, pp. 1-850, or third extended edition, pp. 1-1450 available from URL http://www.physik.fu-berlin.de/ ₹kleinert/b3 See Chapter 5. For a recent application see H. Kleinert, Fluctuation Pressure of Membrane between Walls, Phys. Lett. A 257 , 269 (1999) (cond-mat/9811308).

[14] This will be shown in detail in B. Hamprecht and H. Kleinert (in preparation). Note that in Ref. 11], the authors use even the extrema with the maximal imaginary parts for optimizations which they observe to yield convergence (unfortunately, however, to a wrong result).

[15] See [10, 11, 22] and references cited there.

[16] With standard normalization conditions used in the 3-dimensional $\phi^{4}$-theory, the approach to scaling is governed by Wegner's exponent $\omega$ (see [8]). The present definition of $m_{r}$ differs from the the inverse correlation length $m=\xi^{-1}$ by a factor: $m_{r}=m Z_{\phi}^{-1} \propto m m^{-\eta / 2}$ for $m \rightarrow 0$. This changes the exponent of approach to $\omega^{\prime}=$ $\omega /(1-\eta / 2)$. I thank B. Kastening for noting this.

[17] P. Arnold and G. Moore, Phys. Rev. Lett. 87, 120401 (2001); Phys. Rev. E 64, 066113 (2001). The authors derive a $1 / N$ correction factor $(1-0.527 / N)$ to the leading $N \rightarrow \infty$ result.

[18] V.A. Kashurnikov, N.V. Prokof'ev and B.V. Svistunov, Phys. Rev. Lett. 87, 120402 (2001).

[19] G. Baym, J.-P. Blaizot M. Holzmann, F. Laloë and D. Vautherin, Phys. Rev. Lett. 83, 1703 (1999).

[20] G. Baym, J.-P. Blaizot and J. Zinn-Justin, Europhys. Lett. 49, 150 (2000).

[21] P. Arnold and B. Tomásik, Phys. Rev. A62, 063604 (2000). This paper starts out from the 3+1-dimensional initial theory and derives from it the three-dimensional effective classical field theory, the field-theoretic generalization of the quantum-mechanical effective classical potential of R.P Feynman and H. Kleinert, Phys. Rev. A 34, 5080 (1986). This reduction program was started for the Bose-Einstein gas by A.M.J. Schakel, Int. J. Mod. Phys. B 8, 2021 (1994); J. Mod. Phys. B 8, 2021 (1994); Boulevard of Broken Symmetries, Habilitationsschrift, FU-Berlin, (cond-mat/9805152) (1998). Unfortunately, Schakel did not go beyond the one-loop level so that he was happy to have found a positive shift $\Delta T_{c} / T_{c}$, and did see the cancellation at the two-loop level. See his recent paper Zeta Function Regularization of Infrared Divergences in Bose-Einstein Condensation, (cond-mat/0301050).

[22] F. F. de Souza Cruz, M. B. Pinto and R. O. Ramos, Phys. Rev. B 64, 014515 (2001).

[23] P. Grueter, D. Ceperley, F. Laloe, Phys. Rev. Lett. 79, 3549 (1997) (cond-mat/9707028).

[24] See Eq. (20.23) in the textbook [4] or S.E. Derkachov, J.A. Gracey, and A.N. Manashov, Eur. Phys. J. C 2, 569 (1998) (hep-ph/9705268).

[25] B. Kastening (cond-mat/0303486). 